\title{
The emission of blazars in very-high-energy gamma-rays viewed as a random stationary process: the case of PKS 2155-304
}

\author{
Bernard Degrange*, Giulia Superina, Berrie Giebels and Francesca Volpe ${ }^{\dagger}$ \\ Laboratoire Leprince-Ringuet, École polytechnique \& IN2P3/CNRS \\ E-mail: degrangedllr.in2p3.fr, superinadllr.in2p3.fr, \\ berriedllr.in2p3.fr, volpedllr.in2p3.fr
}

\begin{abstract}
With the advent of the new generation of Cherenkov telescopes such as H.E.S.S., MAGIC, CANGAROO III and VERITAS, about 20 active galactic nuclei belonging to the blazar category have been clearly detected at very high energies. Spectacular flares were observed in a few of them, such as Mkn 421, Mkn 501 and PKS 2155-304, during which gamma-ray fluxes could be sampled with time intervals of a few minutes, an important asset in the quest of a smallest time-scale in the underlying phenomena. In the very-high-energy domain, the experimental situation is however more tricky than in variability studies carried out by X-ray satellites: the time resolution critically depends on the flux itself, due to both photon statistics and background contamination, and the continuous time series are never longer than a few hours. Nevertheless, a description of the observed phenomena by a random stationary process characterized by a simple power density spectrum - a power law of frequency — can be investigated. Such a study requires simulations of very long time series in which the experimental effects are taken into account. Using H.E.S.S. observations of PKS 2155-304 in July 2006, it is shown that different observables, namely excess variances measured over different durations as well as Kolmogorov structure functions, can be consistently accounted for by a simple log-normal process.
\end{abstract}

Workshop on Blazar Variability across the Electromagnetic Spectrum

April 22-25 2008

Palaiseau, France

\footnotetext{
*Speaker.

${ }^{\dagger}$ European Associated Laboratory for Gamma-Ray Astronomy, jointly sipported by CNRS and MPG
} 


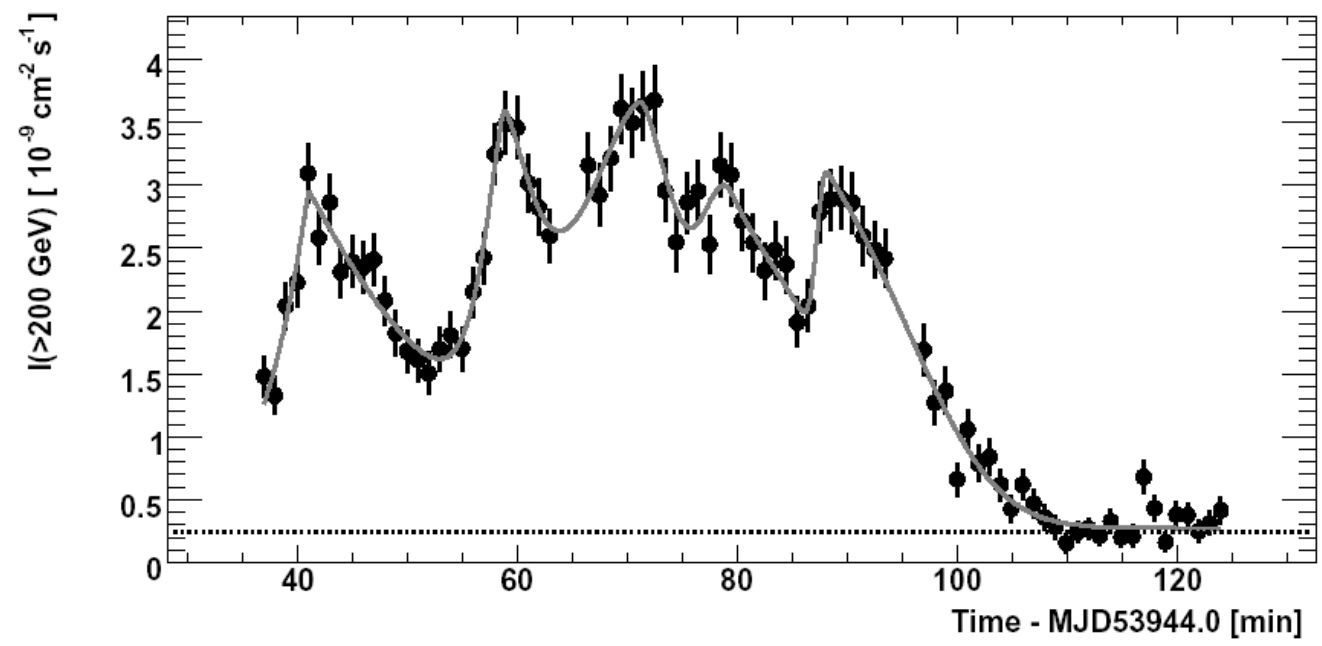

Figure 1: Light curve of the blazar PKS 2155-304 as observed by H.E.S.S. on MJD 53944. The figure is taken from ref. [2].

\section{Introduction}

The recent spectacular increase of the sensitivity of Cherenkov telescopes (H.E.S.S., MAGIC, VERITAS and CANGAROO III) has led to the detection of about 20 blazars emitting in the TeV energy range, as reviewed in this workshop by W. Benbow [1]. Unfortunately, most of these blazars are weak $\mathrm{TeV}$ emitters with fluxes of the order of a few percent of that of the Crab nebula in the same energy range. However, a handful of them (Mkn 421, Mkn 501, 1ES 1959+650 and PKS 2155-304) sometimes exhibit dramatic flares during which the gamma-ray flux is so high that significant variability can be observed, even over timescales of the order of a few minutes (Fig. 1). Until recently, variability studies carried out with Cherenkov telescopes (e.g. [2], [3]) have essentially characterized light curves by empirical variables such as "doubling time", "excess variance", and "fractional variability" per night (see e.g. [4] for definitions).

This situation contrasts with that of the X-ray domain, in which the power spectrum of the fluctuations of the X-ray flux can be measured and is used to define a state of the object. This is the case for galactic X-ray binaries [5] as well as for active galactic nuclei [6]. This is illustrated in Fig. 2 which shows the power spectral density of several X-ray binaries in different states, as a function of frequency. In this framework, it is implicitely assumed that a given state of the system can be described by a random stationary process characterized by the power spectrum, at least during a limited period. In this article, this point of view is applied to the blazar PKS 2155-304, as observed by H.E.S.S. during its flaring period in July 2006 (MJD 53944-MJD 53947).

The experimental constraints limiting the capability of Cherenkov telescopes in variability studies are first reviewed in section 2. Taking these constraints into account, different methods characterizing the source variability are explained in section 3, in particular those in which the power spectral density can be characterized by comparing the experimental light curves to a large sample of very long simulated time series corresponding to a given stationary process. In section 4 , 
BLACK-HOLE-CANDIDATE POWER SPECTRA

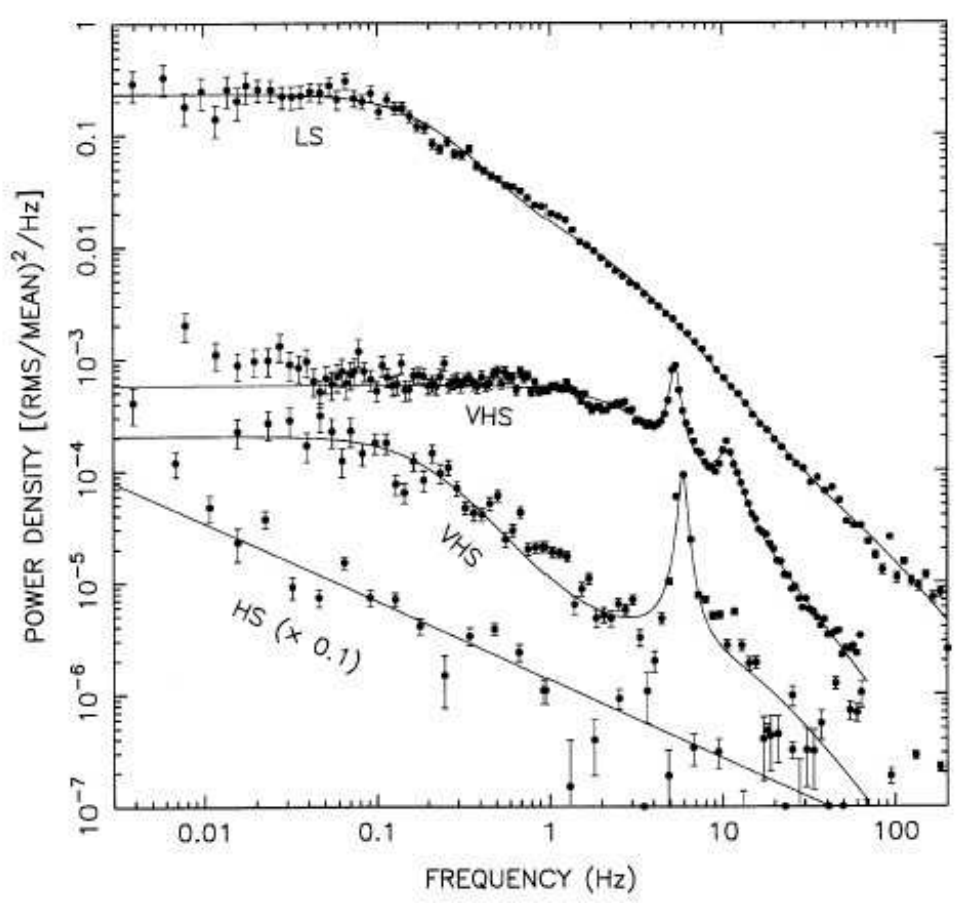

Figure 2: Power spectral density of black hole candidates in X-ray binaries for different states of these objects. The spectrum labeled LS is that of Cyg X1 in the low state. The figure is taken from ref. [5].

such a method is applied to H.E.S.S. data taken on PKS 2155-304 in the period MJD 53944MJD 53947. It is shown that the data can be accounted for by a single logarithmo-normal process and that the power spectral density can be parametrized by a power law of frequency in the interval $\left[10^{-4} \mathrm{~Hz}, 10^{-2} \mathrm{~Hz}\right]$.

\section{Experimental constraints with Cherenkov telescopes}

The high sensitivity of the most recent arrays of Cherenkov telescopes relies on two main factors: firstly, their large effective detection areas, typically $6 \times 10^{4} \mathrm{~m}^{2}$ to $10^{5} \mathrm{~m}^{2}$; secondly, their powerful rejection of the background of hadronic cosmic rays due to fine grain imaging and to stereoscopic reconstruction of atmospheric showers. However, they are submitted to several constraints which make variability studies more tricky than those of satellite experiments:

- Observations require moonless clear nights and generally several sources are observed during the same night; therefore, a given object cannot be continuously monitored during more than a few hours.

- The energy threshold (thus the effective detection area) varies with the zenith angle during the observations due to source tracking. Therefore, spectrum-dependent corrections are necessary to produce a light curve at a fixed energy threshold. 


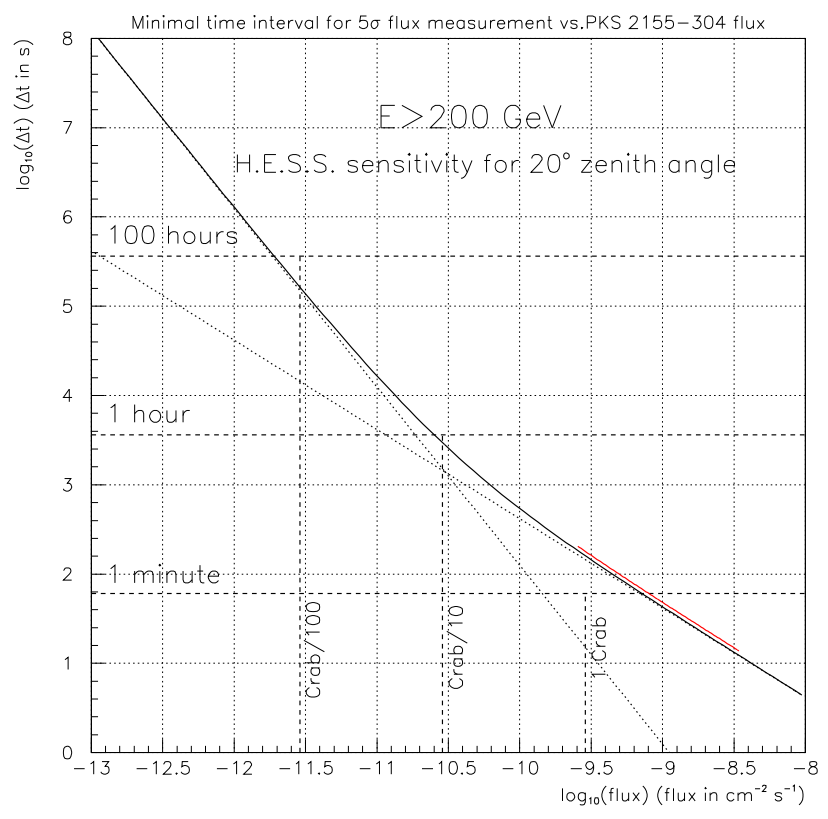

Figure 3: Minimal observation duration for a 5 standard deviation detection of a source by H.E.S.S. at a zenith angle of $20^{\circ}$, as a function of the gamma-ray flux, for an energy threshold of $200 \mathrm{GeV}$. In most observations of PKS 2155-304, the flux was of the order of $10 \%$ of that of the Crab nebula. Fluxes measured during the flares of July 2006 are indicated by the red line superimposed to the curve.

- Finally, the light curve sampling strongly depends on the gamma-ray flux itself. As a consequence, different frequency domains are accessible at different levels of flux.

This last point is illustrated in Fig. 3 which shows the minimal time $\Delta t$ necessary for H.E.S.S. to get a 5 standard deviation detection of a source as a function of the gamma-ray flux above $200 \mathrm{GeV}$ for a $20^{\circ}$ zenith angle. For low fluxes (say less than $10 \%$ that of the Crab nebula), the flux error is dominated by the hadronic background; for higher fluxes, the sensitivity is only limited by photon statistics. These two regimes are indicated by the central dotted lines in Fig. 3. In most of the observations, PKS 2155-304 is found to emit at the level of 10\% of the Crab flux and a significant measurement requires one hour of observation. During the flares of July 2006, the source intensity varied between 1 and more than 10 Crab units, and the figure shows that, in the most favorable situations, it is possible to sample the light curve every minute.

\section{Characterizing the variability}

Several empirical variables have been proposed to characterize the variability of a source. They are the following [4]:

- The "excess variance" $\sigma_{\text {exc }}^{2}$ is obtained by subtracting the contribution of experimental errors from the variance of the fluxes $\left(\Phi_{k}=\Phi\left(t_{k}\right), k=1, N\right)$ measured at different times $t_{k}$ on a 
given light curve segment:

$$
\sigma_{\mathrm{exc}}^{2}=\operatorname{var}(\Phi)-\sigma_{\mathrm{err}}^{2}
$$

- The "fractional variability" $F_{\text {var }}$ is defined as the ratio of the excess r.m.s. $\sigma_{\text {exc }}$ to the average measured flux:

$$
F_{\mathrm{var}}=\frac{\sigma_{\mathrm{exc}}}{\bar{\Phi}}
$$

- The "doubling time" $t_{2}$ is obtained from the fluxes $\Phi_{k}$, in the following way:

$$
t_{2}=\min \left(\tau_{k l}\right) \text { where } \tau_{k l}=\left|\frac{t_{k}-t_{l}}{\Phi_{k}-\Phi_{l}}\right| \frac{\Phi_{k}+\Phi_{l}}{2}
$$

As an alternative definition of the doubling time, one takes the average $t_{2}^{\prime}$ of the 5 smallest values of the $\tau_{k l}$.

All the preceding variables depend on the duration $T$ of the light curve segment over which they are measured, as well as on the size $\Delta t$ of the sampling interval of the light-curve. In our studies, $T$ is hardly larger than a few hours, whereas $\Delta t$ can be of the order of a few minutes during big flares. These figures are quite different from those related to X-ray observations. Moreover, measuring the excess variance (and the corresponding excess r.m.s.) requires that the flux be significant over the light-curve segment of interest. The preceding variables are thus strongly affected by the sensitivity of the instrument and by the intensity of the source which constrain $T$ and $\Delta t$. They are not intrinsic properties of the source.

In contrast, if one adopts the point of view of a random Gaussian stationary process, the power spectral density $P(v)$ as a function of the frequency $v$ is an intrinsic characteristics of the source. As a matter of fact, in the X-ray domain, power spectra are currently measured for binary systems and for active galactic nulei [6]. In the very high energy domain, with short continuous observation durations and with typical measurement errors on the flux, it is delicate to directly determine the power spectrum from the Fourier transform of the light curve since this procedure is very sensitive to windowing effects. On the other hand, power spectra often take the form of a power law of frequency in a large domain of frequencies. Fourier analysis of the PKS 2155-304 light curve of MJD 53944 [2] does suggest such a power law $P(v) \propto v^{-\alpha}$ with an exponent $\alpha$ close to 2 (Fig. 4). Similarly, X-ray observations of PKS 2155-304 by the Beppo-SAX satellite in 1996 and 1997 [4] had also found a power law dependence (Fig. 5) in the same frequency range $\left(10^{-2}-10^{-4} \mathrm{~Hz}\right)$. Such a power density spectrum with an index $\alpha$ close to 2 is that of a red noise, which makes windowing effects particularly important due to low-frequency leakage [7]. In this work, we assume the form of the power law $P(v)=K\left(v_{\text {ref }} / v\right)^{\alpha}$ and give a parametric estimation of the exponent $\alpha$, as well as of the power spectral density $K$ at a reference frequency, namely $v_{\text {ref }}=10^{-4} \mathrm{~Hz}$. This value was chosen to minimize the correlation between the two measured parameters $K$ and $\alpha$. It should be noted that the power law cannot be valid for arbitrarily high or low frequencies. At high frequencies, a cut-off is expected at the smallest time scale imposed by the size of the emitting region (causality). At low frequencies, at least one break is expected to avoid the infrared divergence if $\alpha \geq 1$. The parameters $\alpha$ and $K$ are determined by a maximum likelihood method in which the distributions of the experimental observables are compared to those 


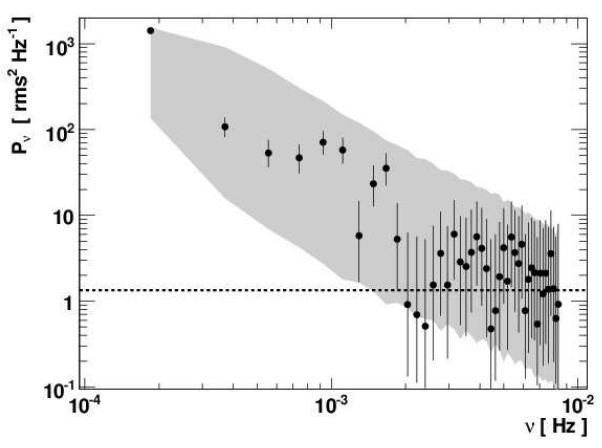

Figure 4: The Fourier power spectrum of the light curve of PKS 2155-304 during 90 minutes on MJD 53944. The dotted line is the level of noise induced by measurement errors. The figure is taken from ref. [2].

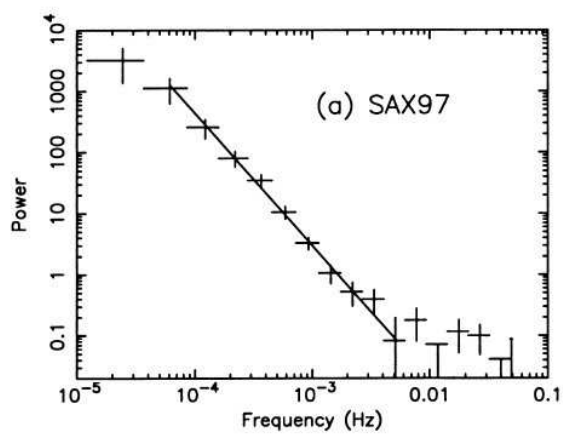

Figure 5: Power spectral density of PKS 2155-304 from X-ray observations by Beppo-SAX in 1997. The figure is taken from ref. [4].

from simulated time series generated by random processes with various values of $\alpha$ and $K$. At this stage, it is important to specify the relevant Gaussian random variable. If the flux results from a sum of different components, it is expected to be Gaussian. On the opposite, if it results from a multiplicative process, its logarithm is expected to be Gaussian [8]. These two possibilities are taken into account in the simulations which are now briefly described.

For practical reasons, simulated light curves are generated by Fourier series, which implies a discrete set of frequencies. This approximation is reasonable if the fundamental frequency is very low. Hence, light-curve segments are extracted from series of duration much longer $\left(\approx 2 \times 10^{5} \mathrm{~s}\right)$ than those of the periods of continuous observations. Each term of the series has a random phase and its Fourier coefficient is normally distributed with 0 mean and with a variance given by the power spectrum. These series are considered to give either the variations of the flux, or those of its logarithm. Simulated light curves are segmented into active periods and gaps of same durations as the experimental ones in order to account for windowing effects. The flux is averaged over time intervals of same duration $\Delta t$ as in the experimental light curve and its value is distorted according to measurement errors in the conditions of real observation. With 500 realistic light curves generated for each set $\{\alpha, K\}$, the expected distributions of any measured variable can then be determined and further used to build likelihood functions.

\section{The exceptional flaring period of PKS 2155-304, as observed by H.E.S.S. in July 2006}

The light curve of PKS 2155-304 during the period of highest intensities (MJD 53944-53947) is shown in Fig. 6 in intervals of $\Delta t=4$ minutes.

\subsection{The excess r.m.s.-flux correlation}

If the gamma-ray flux were the relevant Gaussian variable, its average value would be completely uncorrelated with the higher moments of the light curve, in particular with the excess variance (thus with the excess r.m.s.). On the opposite, a strong r.m.s.-flux correlation is expected if 


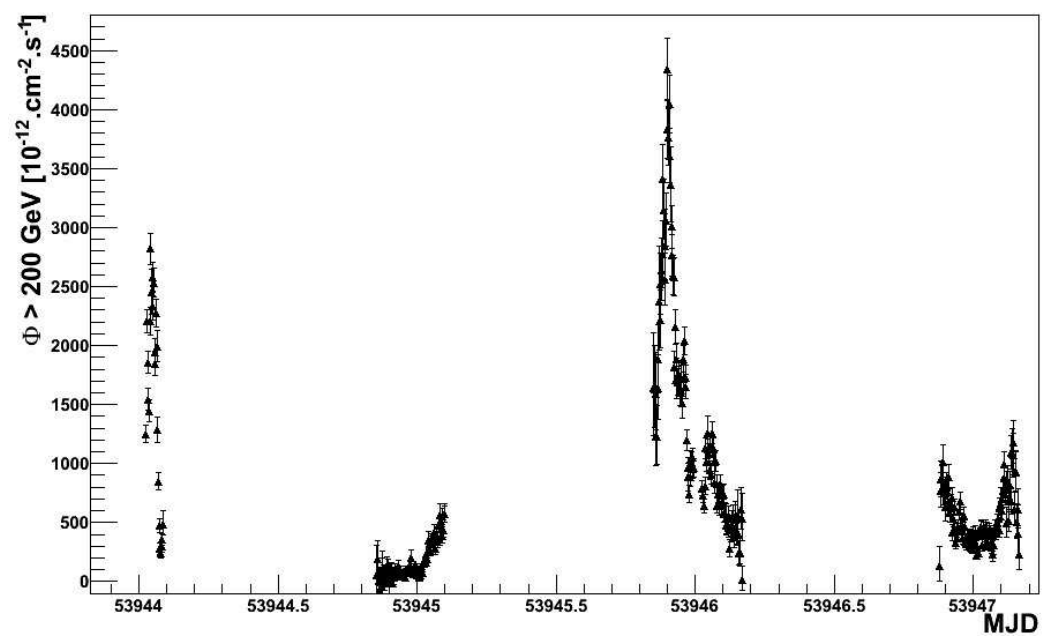

Figure 6: The light curve of PKS 2155-304 as observed by H.E.S.S. during 4 nights: MJD 53944, 53945, 53946 and 53947.

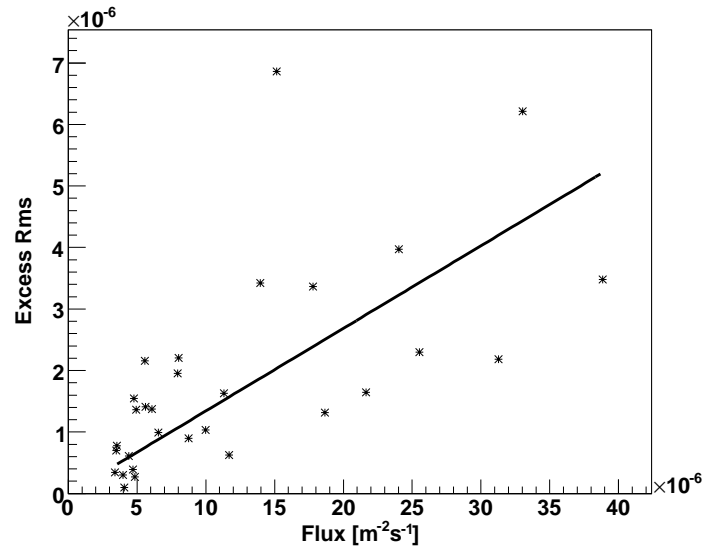

Figure 7: Excess r.m.s. versus average fluxes for segments of light curve of 20 minutes duration, sampled every minute.

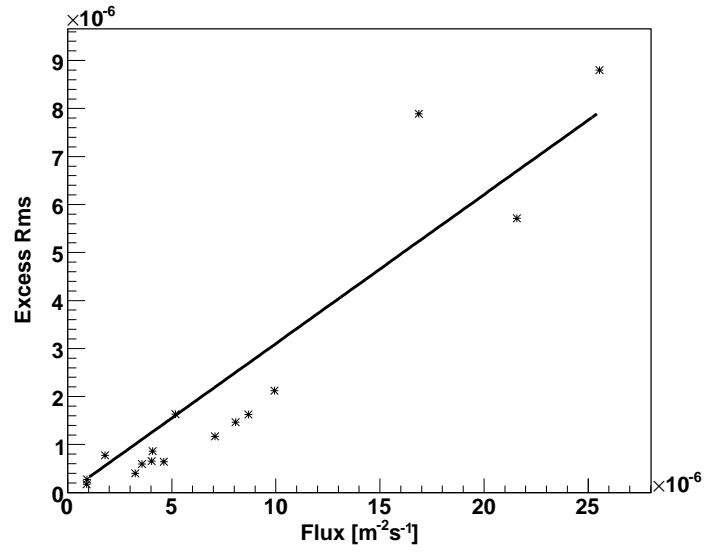

Figure 8: Excess r.m.s. versus average fluxes for segments of light curve of 80 minutes duration, sampled every 4 minutes.

the Gaussian variable is the logarithm of the flux (log-normal process). Such correlations have been observed in the X-ray emission of binary systems and of some active galactic nuclei (see e.g. [8]). In order to investigate a similar correlation in H.E.S.S. data on PKS 2155-304, two kinds of light-curve segments have been used, characterized by their duration $T$ and their sampling interval $\Delta t: T=20$ minutes and $\Delta t=1$ minute for segments of the first kind; $T=80$ minutes and $\Delta t=4$ minutes for segments of the second kind. The excess r.m.s. is plotted versus the average gamma-ray flux in Fig. 7 and in Fig. 8 for segments of the first and of the second kind respectively. With light-curve segments of 20 minutes, the $95 \%$ confidence interval for the correlation coefficient is found to be $[0.36-0.81]$ and is hardly affected when the experimental points in the diagram are moved within the experimental errors on the flux. With light-curve segments of 80 minutes, the correlation coefficient is still higher and found within the interval $[0.84-0.98]$ at $95 \%$ confidence 


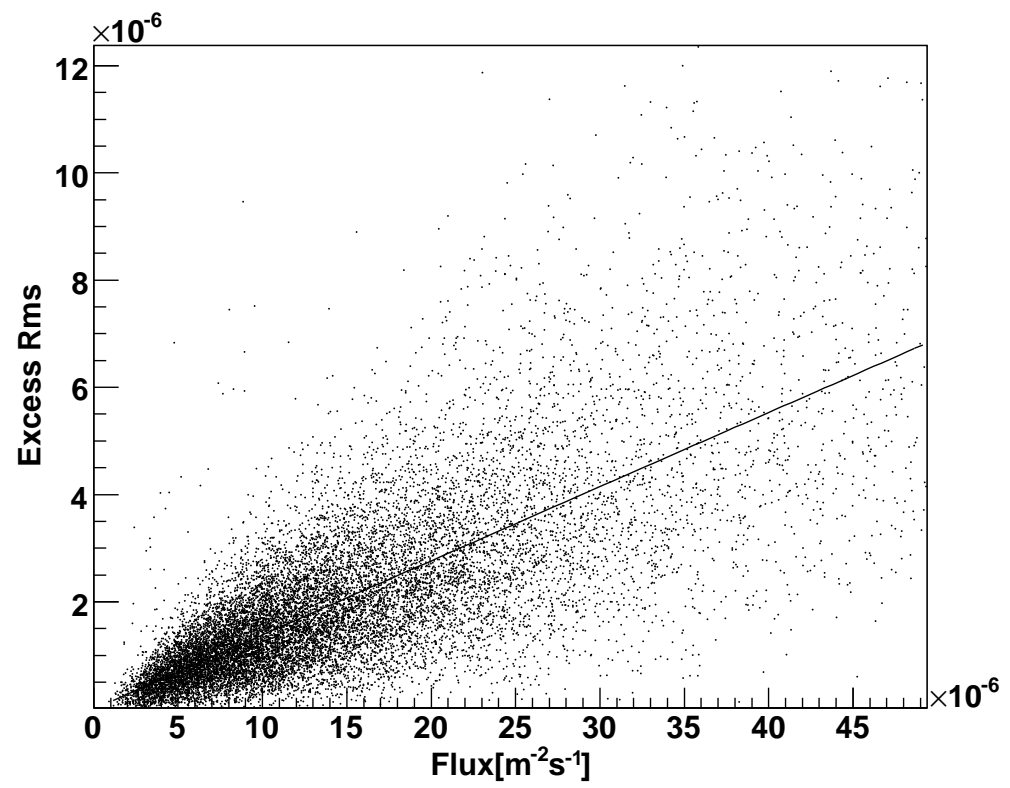

Figure 9: Excess r.m.s. versus average fluxes for 500 simulated segments of light curve of 20 minute duration, sampled every minute, for $\alpha=2$ and $K_{\ell}=500 \mathrm{~Hz}^{-1}$.

level. Here also, the size of experimental errors does not affect the conclusion: the gamma-ray flux is clearly not the relevant Gaussian variable, this possibility being excluded at the level of 7 standard deviations.

Therefore, in the following, data are compared to simulations of log-normal processes characterized by power spectra of the form $K_{\ell}\left(v_{\text {ref }} / v\right)^{\alpha}$. Since the Gaussian variable is $\ln (v)$, the integral of the power spectrum over all frequencies gives the total fractional variability squared which is dimensionless and therefore $K_{\ell}$ is expressed in $\mathrm{Hz}^{-1}$ (or in s). The parameters $\alpha$ and $K_{\ell}$ are then determined by a maximum likelihood method. For each set $\left\{\alpha, K_{\ell}\right\}, 500$ light curves are simulated from which the distributions of the measured quantities are extracted: flux, excess r.m.s. or alternatively $F_{\text {var }}$ for light curve segments of both kinds. This is illustrated in Fig. 9 showing the excess r.m.s.-flux diagram obtained from simulations of a log-normal random process with $\alpha=2$ and $K_{\ell}=500 \mathrm{~Hz}^{-1}$, for light curve segments of 20 minutes. For a given set $\left\{\alpha, K_{\ell}\right\}$, the likelihood function $L\left(\alpha, K_{\ell}\right)$ is built from the simulated distributions of the average flux $\bar{\Phi}$ and from those of the fractional variability $F_{\mathrm{var}}$ measured over light curve segments of a given kind. It should be noted that, for those segments in which the flux does not vary significantly, the calculated excess variance $\sigma_{\text {exc }}^{2}=\operatorname{var}(\Phi)-\sigma_{\text {err }}^{2}$ may be negative so that $F_{\mathrm{var}}=\sigma_{\mathrm{exc}} / \bar{\Phi}$ cannot be calculated. This situation may occur both in data and in simulations in which measurement errors are taken into account. Clearly, the probability of such a situation depends on the parameters $\alpha$ and $K_{\ell}$ and must be taken into account in the maximum likelihood method. Therefore, for a given number of experimental segments (each one yielding an entry in a given histogram), simulations allow to calculate the expectation values of the following quantities:

- number of entries in a given $\bar{\Phi}$ interval; 


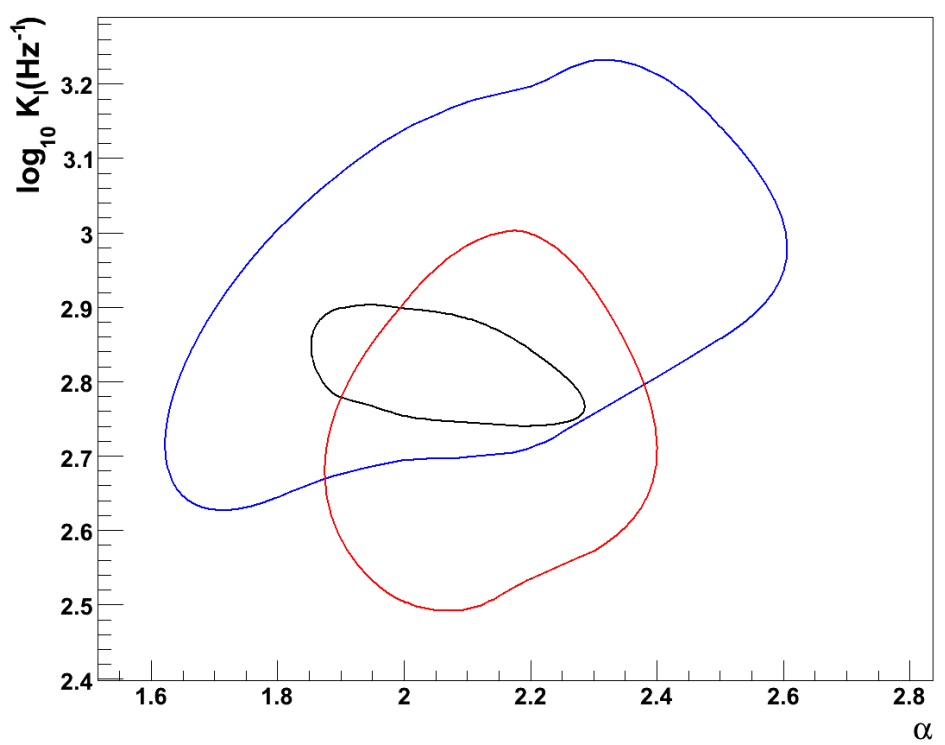

Figure 10: Maximum likelihood determination of $\alpha$ and $K_{\ell}$. Contours are given for $95 \%$ confidence domains in the $\left\{\alpha, K_{\ell}\right\}$ plane. Red contours are obtained by the method explained in section 4.1, using light curve segments of 20 minute duration sampled every minute. Blue contours are similarly obtained with light curve segments of 80 minute duration, sampled every 4 minutes. Black contours are obtained from the study of structure functions explained in section 4.2.

- number of entries in a given $F_{\mathrm{var}}$ interval;

- total number of entries in the last histogram (i.e. those with $\sigma_{\mathrm{exc}}^{2}>0$ ).

The distributions of $\bar{\Phi}$ and $F_{\text {var }}$ are assumed to be independent, which is confirmed by simulations. The likelihood function $L\left(\alpha, K_{\ell}\right)$ is thus given by the product of the probabilities to observe the experimental histograms of $\bar{\Phi}$ and $F_{\text {var }}$, as well as the number of segments with $\sigma_{\mathrm{exc}}^{2}>0$. As a result, 68\% and 95\% confidence regions in the $\left\{\alpha, K_{\ell}\right\}$ plane are shown in Fig. 10. Both kinds of light-curve segments have been used; red contours correspond to $T=20$ minutes and blue contours to $T=80$ minutes. Both yield compatible values of $\alpha$ and $K_{\ell}$. The index $\alpha$ is found to be close to 2, a value similar to those found in previous X-ray observations [4], but the power at $10^{-4} \mathrm{~Hz}$ is higher than the one measured in X-rays by almost an order of magnitude.

\subsection{Structure function}

An alternative way to derive the parameters $\alpha$ and $K_{\ell}$ is provided by the study of the first order Kolmogorov structure function $S(\tau)$ [9] [10], defined as the average value of the squared difference between signals as measured at times separated by a delay $\tau$. Since the relevant Gaussian variable is the logarithm of the flux, the structure function is given by :

$$
S(\tau)=\overline{\left[\ln \Phi\left(t_{k}\right)-\ln \Phi\left(t_{k}+\tau\right)\right]^{2}}
$$




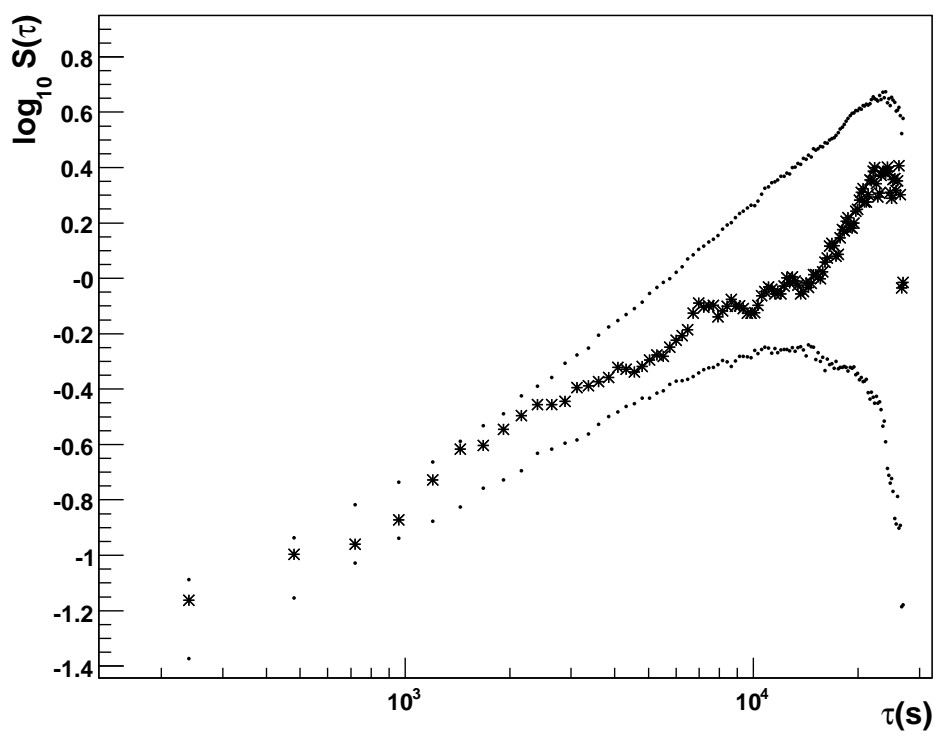

Figure 11: Structure function from PKS 2155-304 observations during 4 consecutive nights MJD 53944MJD 53947 (crosses). The upper and lower dotted lines indicate the limits of a $68 \%$ confidence interval expected from simulations of a statistical ensemble of light curves with $\alpha=2$ and $\log _{10}\left(K_{\ell} / \mathrm{Hz}^{-1}\right)=2.8$.

For small values of $\tau$, this function is essentially equal to twice the variance induced by the measurement errors but at larger values of $\tau$, the function is sensitive to $\alpha$ and $K_{\ell}$. However, large fluctuations from series to series are expected from Monte-Carlo simulations for a given set $\left\{\alpha, K_{\ell}\right\}$. The structure function obtained from the 4 consecutive nights with the highest average intensities (MJD 53944-53947) is given by crosses in Fig. 11. In the same figure, the upper and lower dotted lines indicate the limits of a $68 \%$ confidence interval expected from simulations of a statistical ensemble of light curves with $\alpha=2$ and $\log _{10}\left(K_{\ell} / \mathrm{Hz}^{-1}\right)=2.8$. Moreover, for a fixed value of $\tau$, the distribution of $\ln S(\tau)$ for a given set of parameters $\left\{\alpha, K_{\ell}\right\}$ is found to be almost Gaussian in simulations. Therefore, a likelihood function of $\alpha$ and $K_{\ell}$ based on the first order structure function can be built. The black contours in Fig. 10 show the 95\% confidence region in the $\left\{\alpha, K_{\ell}\right\}$ plane obtained by this method. The result is in good agreement with the one obtained in section 4.1; indeed, the structure function provides a more accurate measurement of $\alpha$ and $K_{\ell}$. All the preceding methods can be combined to give the final result: $\alpha=2.06 \pm 0.21$ and $\log _{10}\left(K_{\ell} / \mathrm{Hz}^{-1}\right)=2.82 \pm 0.08$ at $95 \%$ confidence level.

\subsection{Doubling times}

The doubling times $t_{2}$ and $t_{2}^{\prime}$ defined in section 3 are also often used to characterize the variability of a blazar. They are directly related to the quantities

$$
\tau_{k l}=\left|\frac{t_{k}-t_{l}}{\Phi_{k}-\Phi_{l}}\right| \frac{\Phi_{k}+\Phi_{l}}{2}
$$




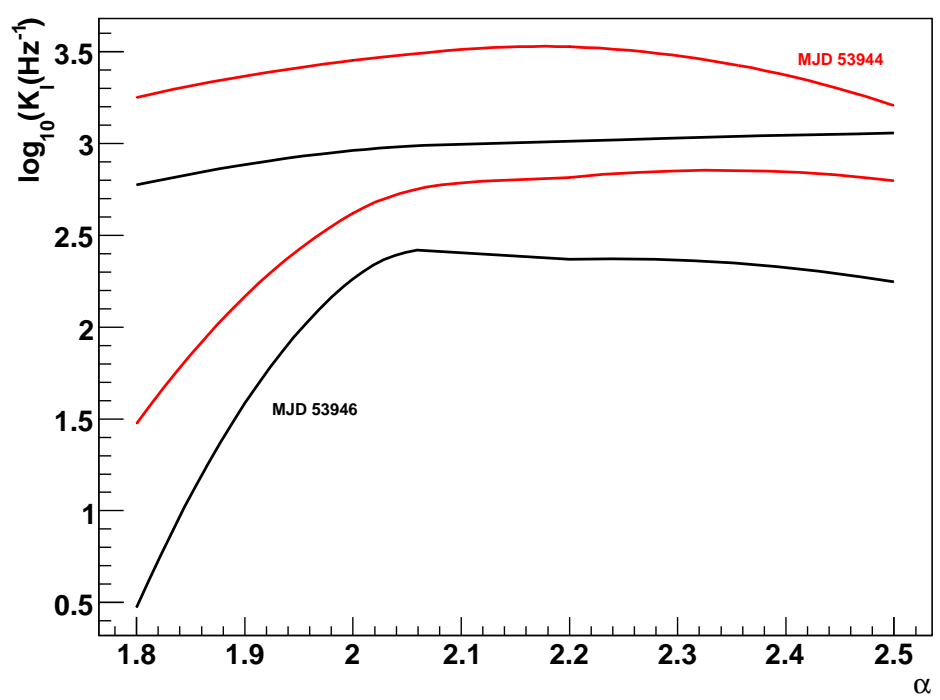

Figure 12: $95 \%$ confidence regions in the $\left\{\alpha, K_{\ell}\right\}$ plane obtained from the doubling times: red contours correspond to MJD 53944 and black ones to MJD 53946.

\begin{tabular}{|c|c|c||c|c|c|}
\hline \multicolumn{3}{|c||}{ MJD 53944 } & \multicolumn{4}{c|}{ MJD 53946 } \\
\hline \multicolumn{1}{|c|}{$t_{2}=\min \left(\tau_{k l}\right)$} & $t_{2}^{\prime}=\overline{\left(5 \text { smallest } \tau_{k l}\right)}$ & $\Delta t$ & $t_{2}=\min \left(\tau_{k l}\right)$ & $t_{2}^{\prime}=\overline{\left(5 \text { smallest } \tau_{k l}\right)}$ \\
\hline 1 min. & $1.28 \pm 0.38$ & $2.84 \pm 1.00$ & 1 min. & $1.38 \pm 0.37$ & $2.33 \pm 0.97$ \\
\hline 2 min. & $2.63 \pm 0.54$ & $4.53 \pm 1.59$ & 2 min. & $3.49 \pm 1.00$ & $6.22 \pm 2.22$ \\
\hline
\end{tabular}

Table 1: Doubling times $t_{2}$ and $t_{2}^{\prime}$ in minutes from two observation periods of PKS 2155-304 (MJD 53944 and MJD 53946) with different sampling intervals. See text for the definitions of $t_{2}$ and $t_{2}^{\prime}$.

defined for each pair of flux measurements in the light curve. In the determination of $t_{2}=\min \left(\tau_{k l}\right)$ or $t_{2}^{\prime}$ (average of the 5 smallest values of $\tau_{k l}$ ), pairs are taken into account only if they satisfied the two following conditions:

- both fluxes $\Phi_{k}$ and $\Phi_{l}$ must be different from zero with a significance greater than 2 standard deviations;

- the relative error on the flux difference $\left|\Phi_{k}-\Phi_{l}\right|$ must be smaller than $30 \%$.

However $t_{2}$ and $t_{2}^{\prime}$ strongly depend on the sampling interval $\Delta t$ of the light curve as shown in Table 1 for two data sets (MJD 53944 and MJD 53946). Simulations can also be used to investigate the sensitivity of the doubling times to the parameters $\alpha$ and $K_{\ell}$. The $95 \%$ confidence regions in the $\left\{\alpha, K_{\ell}\right\}$ plane obtained from doubling times are shown in Fig. 12; these regions only constrain $\log _{10}\left(K_{\ell}\right)$ for $\alpha>2$ and yield values compatible with the result given in section 4.2. On the other hand, doubling times are absolutely not sensitive to $\alpha$.

Doubling times are often quoted to set an upper limit on the ultimate causality time scale in the gamma-ray emission process. However, it can be seen from Table 1 that their values are close to 
the minimal time to get a 5 standard deviation significance of the signal at high flux. Therefore, in the future, more sensitive arrays of Cherenkov telescopes are likely to find smaller doubling times. This can be checked by assuming no high-frequency cutoff in the spectrum and by simulating a blazar flare with $\alpha=2, \log _{10}\left(K_{\ell} / \mathrm{Hz}^{-1}\right)=2.8$ and an intensity comparable to that of PKS 2155304 on MJD 53946, as observed by a square array of 36 telescopes such as those of H.E.S.S. I. It turns out that the light curve can then be sampled in intervals of $20 \mathrm{~s}$ and that the doubling time $t_{2}$ is found to be $25 \mathrm{~s}$ (with $30 \%$ accuracy). Only if a significantly larger value of $t_{2}$ were found, could one claim a real cutoff in the power spectrum related to the size of the emitting region.

\section{Conclusion}

The variability of PKS 2155-304 in very-high-energy gamma-rays, as observed by H.E.S.S. during the flaring period in July 2006, has been investigated as a random stationary process by means of several methods which all yield consistent results. This study is the first of its kind carried out on a blazar at very high energies. The observations can be well described by a log-normal random process, which suggests an underlying multiplicative phenomenon. Between $10^{-4} \mathrm{~Hz}$ and $10^{-2} \mathrm{~Hz}$, the power spectrum can be described by a power law with the index $\alpha=2.06 \pm 0.21$, typical of a red noise; the power $K_{\ell}$ at $10^{-4} \mathrm{~Hz}$ is given by $\log _{10}\left(K_{\ell} / \mathrm{Hz}^{-1}\right)=2.82 \pm 0.08$. A similar red noise was observed in the X-ray emission of PKS 2155-304, but with a much lower X-ray power at $10^{-4} \mathrm{~Hz}$. The preceding study should now be completed in several ways. First, it is important to correlate the power density spectrum with the energy spectrum, in particular to investigate a variation of the parameters in different energy bands. It may help characterize the different states of the blazar. It is also important to investigate the power spectrum at lower frequencies, looking for a spectral break which is necessary to avoid infrared divergence. New H.E.S.S. observations and forthcoming multi-wavelength campaigns will be particularly useful to achieve this program.

\section{References}

[1] W. Benbow, in proceedings of Workshop on Blazar Variability across the Electromagnetic Spectrum, Palaiseau, France (2008).

[2] F. Aharonian, et al., ApJ 664 (2007) L71.

[3] J. Albert, et al., ApJ 669 (2007) 862.

[4] Y. H. Zhang, et al., ApJ 527 (1999) 719.

[5] M. Van der Klis, in X-ray Binaries, W.H.G. Lewin et al. (eds.), Cambridge Univ. Press, p. 252, 1995.

[6] S. Vaughan, et al., MNRAS 345 (2003) 1271.

[7] J. Deeter, ApJ 281 (1984) 482.

[8] P. Uttley, et al., MNRAS 359 (2005) 345.

[9] W.C. Lindsey \& C.M. Chie, Proc. IEEE 64 (1976) 1652.

[10] J. Rutman, Proc. IEEE 66 (1978) 1048. 